\title{
Modeling the mitogen activated protein (MAP)-kinase pathway using ordinary differential equations
}

\author{
Sharadwata Pan*, Sameer E. Mhatre \\ Department of Chemical Engineering, Indian Institute of Technology Bombay, Powai, Mumbai - 400076, India
}

Email address:

sharadwata@gmail.com(S. Pan), sameer.e.mhatre@gmail.com(S. E. Mhatre)

\section{To cite this article}

Sharadwata Pan, Sameer E. Mhatre. Modeling the Mitogen Activated Protein (MAP)-Kinase Pathway Using Ordinary Differential Equations. Computational Biology and Bioinformatics. Vol. 1, No. 2, 2013, pp. 6-9. doi: 10.11648/j.cbb.20130102.11

\begin{abstract}
Mitogen Activated Protein (MAP) Kinase pathway is central to comprehend the key cellular signal transduction mechanisms in animal physiology, including human beings. Modeling MAP Kinase pathway has two main applications: deciphering a transient response of three main components (MAPKKK, MAPKK and MAPK) to regulate signaling and utilizing the models to make it behave as a potential drug target. The current study develops a mathematical representation for this transient cell-signaling pathway, based on a simple modular approach to structure and model a three-tier cascade. Based on assumptions from existing literature, ordinary differential equations have been formulated to express the concentrations of MAPKKK, MAPKK and MAPK as a function of time. Finally, the transient responses of the deduced concentrations of these components are analyzed to understand the pathway behavior and some interesting results are obtained by analyzing the temporal evolutions of the concentrations of the six components involved in the pathway. We conclude that the transient behaviour of MAPKKK can be captured with first order ordinary differential equations. Some anomalous behavior is observed in case of MAPKK and MAPK. In this perspective, future work can be designed to regulate the MAP Kinase signaling by taking Michaeli-Menten kinetics into consideration and make complex models to get more accurate results.
\end{abstract}

Keywords: Modeling, MAP-Kinase, MAPKKK, MAPKK, MAPKK, Cascade, Differential Equation

\section{Introduction}

In the signal transduction mechanism of humans, Mitogen Activated Protein (MAP)-Kinase pathway is an important modular unit composed of three kinases: MAP-Kinase Kinase Kinase (MAPKKK), MAP-Kinase Kinase (MAPKK) and MAP-Kinase (MAPK). Upon sequential phosphorylation with each other, these kinases are activated in response to a varied range of external and internal stimuli, such as cellular stress, growth factors, cell adherence, cytokines and neurotransmitters[1]. With one of the most basic signaling designs found in biological signal transduction system, the MAP-K pathway is represented by a cycle formed by a kinase (which phosphorylates a target protein) and a contrasting phosphatase (which dephosphorylates the target)[2]. In the MAP-K pathway, majority of the activators commence signaling by activating the receptors in the cell membrane, which in turn accumulate into receptor signaling complexes and turn on a MAPKKK typically through the action of a small GTPase[3]. Within the MAP-K unit, transduction of signals is reasonably specific and is habitually identified with linearity in its basic structure. The quantity of known MAPK effectors, however, is very large and varied, including mainly cytoskeletal proteins, protein kinases and transcription factors. When activated, MAPK gets translocated to the nucleus from cytoplasm, where it controls transcription of genes by modifying transcription factor activity and changing the chromatin structure. There are at least 8 MAPKs, 7 MAPKKs and 12 different MAPKKKs in the eukaryotic cells, which can be credited to no less than four functionally dissimilar MAPK components[2].

Huang and Ferrell proposed the first model in 1996[4] where they revealed the ultrasensitivity of the signaling cascade. In 1997, two models were proposed in quick succession[2] that showed a two-collision, distributive mechanism for the activating dual phosphorylation of MAP itself. It suggested that after phosphorylating the first site, MEK dissociates and then again rebinds to add a phosphate to the second site. An escalating number of MAP-K cascade models have been developed (summarized in[2]) in the last decade that evolved in both complexity and size over the years[1-4]. 


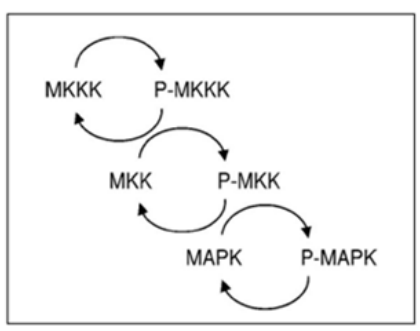

(a)

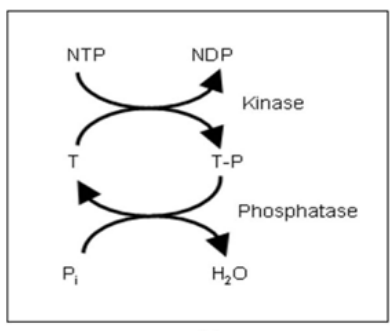

(b)
Figure 1. (a) Three-tier MAP Kinase Cascade (reproduced from[2]). (b): Mechanism of action through phosphorylation (reproduced from [3])

The motivation for the current study was two-fold: the vast range of applications of the MAP-K pathway and progress in modeling affected by overpowering complexity rather than a simple modular conceptualization of the signaling cascade. In standard Molecular Biotechnology, the MAP-K pathway is perhaps the most rigorously studied pathway[1, 5]. The pathway is also vital, owing to its regulation of the growth, proliferation, differentiation and apoptosis (cell-death) of major cell types[6]. The MAP-K pathway can also be modeled to capture the in vivo metabolic complexities associated with abnormal cellular growth such as cancer[2]. It may also act as a suitable drug target[2]. It is therefore scientifically important and challenging to model this pathway and get a better understanding of all these cellular machineries. The innate challenge stems from the involvement of a wide array of transcription factors (Grb2, Ras, ERK, MEK etc.), each of which may generate sub-cascades. This intricacy, in turn, acts as the initial 'substrate' for stochastic and ODE modeling.

The outline of the paper is as follows. In the next section, the MAP-K cascade is visualized as a three-tier cascade, structured and ODEs are formulated with appropriate assumptions / boundary conditions. Section 3 contains details about solving the ODEs and a discussion about the steady state and temporal evolutions of the concentrations of the six components involved in the pathway. The conclusions from the current work are summarized in Section 4.

\section{Methodology}

\subsection{General MAP-Kinase Cascade and Assumptions}

The general layout of the MAPK pathway consists of three kinases / phosphatase cycles built into a three-tiered cascade (see Figure 1(a)), consisting of a MAPK, which is activated via phosphorylation (see Figure 1(b)), by a MAPKK / MKK (MAPK kinase), which in turn is phosphorylated by a MAPKKK / MKKK (MAPKK kinase).

MAPKs are deactivated by a family of phosphatases termed MKPs (MAPK phosphatases)[2]. It is now well known that the linear signaling MAP-K cascades are comparatively easier to model which may give $n$-th order response[8], but accurate quantification of response in highly unlikely. This signifies the need for a branched network where the rate law and chemical kinetics have to be followed, which is the crux of the methodology for the current study. Also, it has been suggested to identify the key elements in a MAP-K cascade so that the main properties can be reinstated without modeling all the biological entities[7]. In other words, we may overlook compartmentalization while assuming a simpler cascade. Hence, in this study, we have considered only the main components, MAPK, MAPKK, MAPKKK and phosphokinase (PK), phosphatase (PP). Also, while modeling, the need for easier understanding of the signaling pathway is expected by maintaining individuality while defining symbols and parameters[9]. For this, we have used ' $\mathrm{K}$ (odd)' to denote phosphorylation and ' $\mathrm{K}$ (even)' to denote dephosphorylation. Please note that the letter ' $\mathrm{K}$ ' may either be in upper or lower case, as applicable.

\subsection{Structuring and Modeling the Map-Kinase Cascade}

\subsubsection{Schematic Representation}

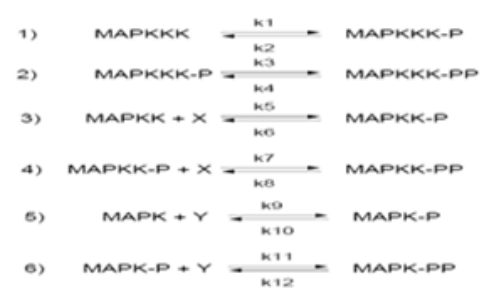

Based on assumptions from past studies[7 - 9], we are proposing a simplified three-tiered cascade of the MAP Kinase Pathway, which is shown in Figure 2. From the above cascade, based on the assumptions we have made, we can deduce the above reversible reactions (reactions 1 to 6 ).

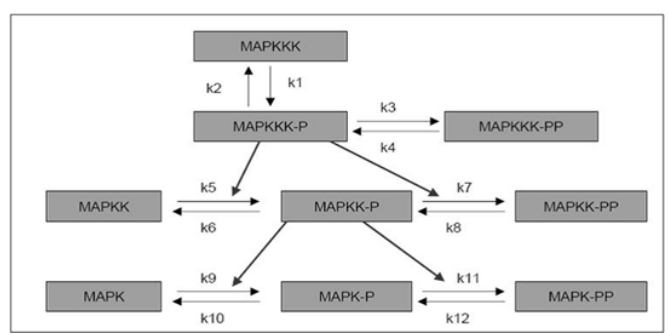

Figure 2. A simplified three-tiered cascade of the MAP-Kinase Pathway. Main components of the proposed structure: MAPKKK: Map Kinase Kinase Kinase; MAPKK: Map Kinase Kinase; MAPK: Map Kinase; MAPKKK-P: Map Kinase Kinase Kinase (Phosphorylated); MAPKK-P: Map Kinase Kinase (Phosphorylated); MAPK-P: Map Kinase (Phosphorylated); phosphorylation: forward reaction; dephosphorylation: backward reaction; $k 1, k 3, k 5, \ldots \ldots \ldots . ., k 11$ : forward rate constants; $k 2, k 4$, $k 6, \ldots \ldots \ldots . ., k 12$ : backward rate constants.

\subsubsection{Ordinary Differential Equation (ODE) Representation of the Cascade}

For MAPKKK: Here we are assuming that rate of change of[MAPKKK] will depend upon[MAPKKK] and[MAPKKK-P]. 


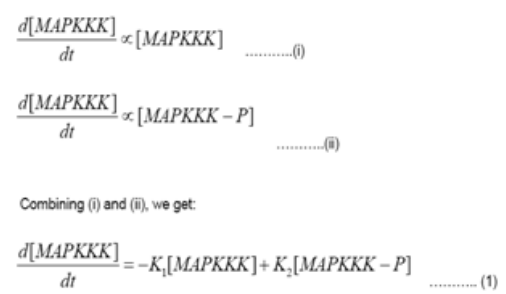

For MAPKKK-P (Phosphorylated MAPKKK): Here we are assuming that rate of change of[MAPKKK-P] will depend upon[MAPKKK-P] and[MAPKKK-PP].

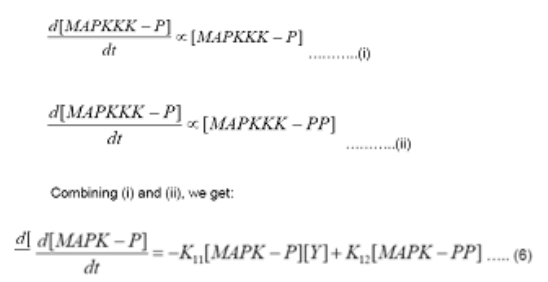

Similarly, we have derived differential equations for MAPKK, MAPKK-P, MAPK and MAPK-P. At a glance, all the equations are given below:

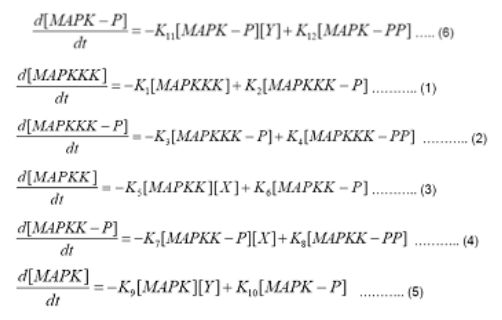

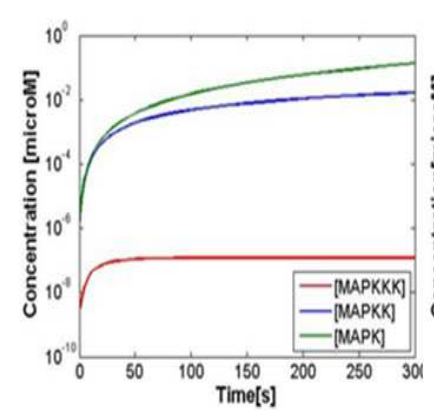

(a)

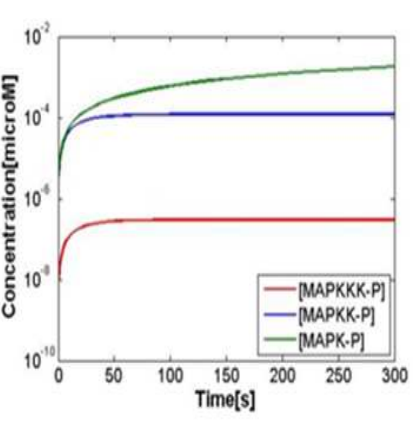

(b)
Figure 3. Transient responses for (a) unphosphorylated and (b) phosphorylated MAPKKK, MAPKK and MAPK. The onset of steady state is occurs fairly early for both phosphorylated and unphosphorylated $M A P K K K$ and $M A P K K$, and late for MAPK.

\section{Results and Discussion}

\subsection{Solving the ODEs}

Here we are assuming[MAPKKK-PP] to be a constant, $\mathrm{C}_{1}$. Also, all the equations are linear first order non-homogeneous differential equations. So the Integrating Factor method of solution has been followed. Considering all these, the ODEs for MAPKKK can be written as:

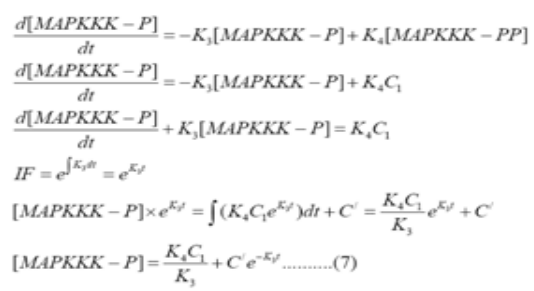

Putting (7) in (1) we get:

$$
\begin{aligned}
& \frac{d[\text { MAPKKK }]}{d t}+K_{1}[\text { MAPKKK }]=\frac{K_{2} K_{4} C_{1}}{K_{3}}+K_{2} C e^{-K_{1} t} \\
& I F=e^{j R_{\text {ite }}}=e^{K_{f}}
\end{aligned}
$$

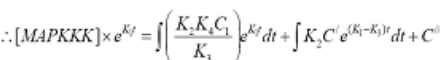

$$
\begin{aligned}
& \Rightarrow[M A P K K K] \times e^{K_{1 t}}=\frac{K_{4} C_{1} K_{2}}{K_{3} K_{1}} e^{K_{f}}+\frac{K_{2} C^{\prime}}{K_{1}-K_{3}} e^{\left(X_{1}-K_{j}\right) \prime}+C^{\prime \prime} \\
& \Rightarrow[M A P K K K]=\frac{K_{4} C_{1} K_{2}}{K_{3} K_{1}}+\frac{K_{2} C}{K_{1}-K_{3}} e^{-K_{K_{t}}}+C^{y} e^{-K_{f}} \ldots \ldots(a)
\end{aligned}
$$

Proceeding the same way, we have derived expressions for MAPKK and MAPK. At a glance, the expressions for

$$
\begin{aligned}
& {[M A P K K K]=\frac{K_{4} C_{1} K_{2}}{K_{3} K_{1}}+\frac{K_{2} C^{\prime}}{K_{1}-K_{3}} e^{-K_{,} \prime}+C^{\prime \prime} e^{-K_{y} \prime} \ldots \ldots . .(a)}
\end{aligned}
$$

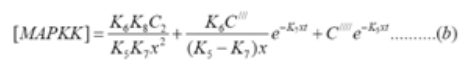

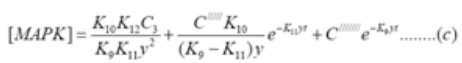

MAPKKK, MAPKK and MAPK are shown in (a)-(c).

Here, C'- C',",' are constants. Now, in order to solve C', C", C", C",", C"," and C",",, we have used the initial conditions from an earlier work by Huang et al.[4]: At $\mathrm{t}=$ $0,[\mathrm{MAPKKK}]=0.003 \mu \mathrm{M},[\mathrm{MAPKK}]=1.2 \mu \mathrm{M},[\mathrm{MAPK}]=$ $1.2 \mu \mathrm{M}$. Also, at $\mathrm{t}=0$,[MAPKKK-P $]=0$,[MAPKK-P $]=$ $0,[\mathrm{MAPK}-\mathrm{P}]=0$. Using initial conditions, finally the main components may be expressed as:

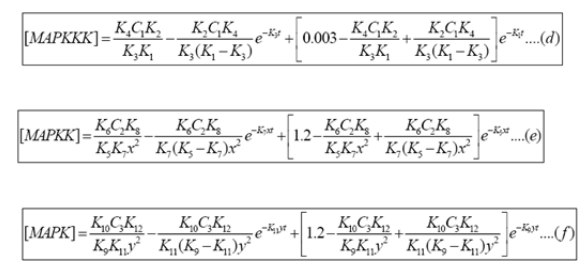

\subsection{Transient Response and Steady State Behavior}

In order to solve the equations (d), (e) and (f), we have taken the values of forward and backward rate constants from elsewhere[4]. For phosphorylation of MAPKKK, K1 = $0.5 \mathrm{~s}^{-1}$, phosphorylation of MAPKKK-P, K3 $=0.05 \mathrm{~s}^{-1}$, phosphorylation of MAPKK, K5 $=0.5 \mathrm{~s}^{-1}$, phosphorylation of MAPKK-P, K7 $=0.05 \mathrm{~s}^{-1}$, phosphorylation of MAPK, K9 $=2 \mathrm{~s}^{-1}$, phosphorylation of MAPK-P, K11 $=0.2 \mathrm{~s}^{-1}$. For dephosphorylation of all the above, the rate constants (K2, $\mathrm{K} 4, \mathrm{~K} 6, \mathrm{~K} 8, \mathrm{~K} 10$ and $\mathrm{K} 12$ ) are taken to be $0.5 \mathrm{~s}^{-1}$ [4]. In addition, we have guessed: $\mathrm{C} 1=0.03 \mu \mathrm{M}, \mathrm{C} 2=12 \mu \mathrm{M}$ and $\mathrm{C} 3=12 \mu \mathrm{M}$. Also, $\mathrm{X}$ (at $\mathrm{t}=100 \mathrm{~ms}) \approx 0.003 \mu \mathrm{M}$; Y (at $\mathrm{t}=$ $100 \mathrm{~ms}) \approx 0.001 \mu \mathrm{M}$. The assumptions are that $\mathrm{C} 1, \mathrm{C} 2$ and $\mathrm{C} 3$ values are ten times (10X) the initial values of MAPKKK, MAPKK and MAPK. The values of $\mathrm{X}$ and $\mathrm{Y}$ have been 
calculated at 100 milliseconds after the pathway starts functioning. After taking several different values of time and using equations (d), (e) and (f), we derived transient responses for unphosphorylated and phosphorylated MAPKKK, MAPKK and MAPK (see Figure 3). It is clear that the onset of steady state occurs fairly early for both phosphorylated and unphosphorylated MAPKKK. The steady state is achieved fairly early for phosphorylated MAPKK, but for the unphosphorylated MAPKK, the concentration demonstrates an increasing trend with time, for a prolonged period. It's not clear exactly when the concentration becomes independent of time. However, for both phosphorylated and unphosphorylated MAPK, there is an increasing trend for concentration versus time. This is somewhat arguable, and may need further examination. So, the degree or extent of phosphorylation does seem to affect the achievement of steady state for MAPKKK and MAPKK, and not influence the achievement of steady state for MAPKKK and MAPKK, and not influence the achievement of steady state for MAPK.

\section{Conclusions}

A generalized and simplified MAP Kinase cascade has been structured. Based on assumptions from past studies, linear non-homogeneous first order differential equations were formulated based on the reactions. MAPKKK, MAPKK and MAPK concentrations were expressed as a function of time. Assuming some arbitrary values and some values from existing studies, a plot was created between the concentrations and time. Though MAPKKK behaved in sync with theoretical predictions, but some anomalous behavior was observed in case of MAPKK and MAPK. Future works can be carried out by incorporating Michaeli-Menten kinetics into modeling and analysis of complex cascading simulations.

\section{Acknowledgements}

The authors would like to thank Prof. S. Arunkumar at IIT Bombay (India) for insightful discussions and suggestions and Sanketkumar V. Solanki and Nishu Goyal for modeling assistance.

\section{Author Disclosure Statement}

No competing financial interests exist.

\section{References}

[1] Cobb, M. H.: MAP Kinase Pathways; Prog. Biophys. Mol. Biol.; vol. 71, pp. 479-500. (1999)

[2] Orton, R. J., Sturm, O. E., Vyshemirsky, V., Calder, M., Gilbert, D. R., and Kolch, W.: Computational modelling of the receptor-tyrosine-kinase-activated MAPK pathway; Biochem. J.; vol. 392; pp. 249-261.(2005)

[3] Alberts, B., Johnson, A., Lewis, J., Raff, M., Roberts, K., and Walter, P.: Molecular Biology of the Cell (1st edition), Chapter 15, Garland Science: New York. p. 948. (1982)

[4] Huang, C. Y. F., and Ferrell (Jr.), J. E.: Ultrasensitivity in the mitogen-activated protein kinase cascade; Proc. Natl. Acad. Sci.; vol. 93; pp. 10078-10083.(1996)

[5] Widmann, C., Gibson, S., Jarpe, M. B., and Johnson, G. L.: Mitogen-Activated Protein Kinase: Conservation of a Three-Kinase Module From Yeast to Human; Physiol. Rev.; vol. 79; pp. 143-180.(1999)

[6] Chang, L., and Karin, M.: Mammalian MAP kinase signalling cascades; Nature (London); vol. 410; pp. 37-40.(2001)

[7] Rodriguez, J. S., Kremling, A., Conzelmann, H., Bettenbrock, K., and Gilles, E. D.: Modular Analysis of Signal Transduction Networks (Feature Article); IEEE Control Systems Magazine; vol. 24; pp. 35-52.(2004)

[8] Hornberg, J. J., Heinrich, R., Bruggeman, F. J., Binder, B., Geest, C. R., Marjolein Bij de Vaate, A. J., Lankelma, J., and Westerhoff, H.V.: Principles behind the multifarious control of signal transduction; FEBS Journal; vol. 272; pp. 244-258.(2005)

[9] Schoeberl, B., Eichler-Jonsson, C., Gilles, E. D., and Muller, G.: Computational modeling of the dynamics of the MAP kinase cascade activated by surface and internalized EGF receptors; Nat. Biotechnol.; vol. 20; pp. 370-375.(2002) 